\title{
Rationale and Design for a Study Using Intravascular Ultrasound to Evaluate Effects of Rosuvastatin on Coronary Artery Atheroma in Japanese Subjects COSMOS Study (Coronary Atherosclerosis Study Measuring Effects of Rosuvastatin Using Intravascular Ultrasound in Japanese Subjects)
}

\author{
Tadateru Takayama, MD; Takafumi Hiro, MD*; Masakazu Yamagishi, MD**; \\ Hiroyuki Daida, MD ; Satoshi Saito, MD ${ }^{\dagger+}$; \\ Tetsu Yamaguchi, MD; Masunori Matsuzaki, MD*
}

\begin{abstract}
Background There have been few multicenter studies using intravascular ultrasound (IVUS) to assess the process of atherosclerosis in a Japanese population with hypercholesterolemia that is being treated with 3hydroxy-3-methylglutaryl coenzyme A reductase inhibitors for control of low-density lipoprotein-cholesterol. Methods and Results An open-label multicenter study is planned to evaluate with IVUS whether treatment with rosuvastatin for 76 weeks results in regression of coronary artery atheroma volume in patients who have coronary heart disease (CHD) and hypercholesterolemia. Sample size is 200 subjects with CHD who are to undergo percutaneous coronary intervention. The planned duration is between October 2005 and October 2008. Conclusions The COSMOS study will be the first multicenter cardiovascular study in a Japanese population and may provide new evidence on the effects of rosuvastatin on the progression of coronary atherosclerotic lesions. (Circ J 2007; 71: 271-275)
\end{abstract}

Key Words: Atherosclerosis; Coronary disease; Intravascular ultrasound; Lipids; Rosuvastatin

C oronary heart disease (CHD) is the single largest cause of death of men and women in many countries. The Framingham Heart Study identified total cholesterol (TC) as a major contributor to CHD and strongly related to progression of the disease 1,2 The National Cholesterol Education Program Adult Treatment Panel II (NCEP ATP II) identified low-density lipoprotein (LDL)cholesterol (C) as the primary target for cholesterol-lowering therapy to prevent CHD (NCEP ATP II 1993)? NCEP ATP III clinical updates include guidelines recommending intensive dietary and drug management of LDL-C in patients with CHD (ATP II) and more intensive LDL-lowering therapy for high-risk patients (ATP III) in order to achieve LDL-C levels $<100 \mathrm{mg} / \mathrm{dl}[2.59 \mathrm{mmol} / \mathrm{L}]$ (NCEP ATP III 2001)4. A high LDL-C level is recognized as an indepen-

(Received August 1, 2006; revised manuscript received October 19 , 2006; accepted November 9, 2006)

Division of Cardiovascular Medicine, Department of Medicine, Nihon University School of Medicine, Tokyo, *Division of Cardiology Medicine, Department of Medicine and Clinical Science, Yamaguchi University Graduate School of Medicine, Ube, **Division of Cardiovascular Medicine, Graduate School of Medical Science, Kanazawa University, Kanazawa, Department of Cardiology, Juntendo University School of Medicine, Hepartment of Advanced Medicine, Nihon University School of Medicine and Cardiovascular Center, Toranomon Hospital, Tokyo, Japan

Mailing address: Satoshi Saito, MD, Department of Advanced Medicine, Nihon University School of Medicine, 30-1 Ohyaguchikami-cho, Itabashi-ku, Tokyo 173-8610, Japan. E-mail: satoshis@med.nihon-u. ac.jp dent risk factor for CHD events and many guidelines therefore advocate LDL-C reduction. The Japan Lipid Intervention Trial (J-LIT), which is a national cohort study, showed that normalization of the lipid concentration reduced the risk of coronary events in 52,421 Japanese patients with hypercholesterolemia?

Statins are now the most widely used medication for the treatment of hypercholesterolemia because they partially inhibit 3-hydroxy-3-methylglutaryl coenzyme A (HMG-CoA) reductase, which is the rate-limiting step in cholesterol synthesis. HMG-CoA reductase inhibition consequently induces the compensatory upregulation of hepatic LDL receptors, which enhances the LDL-C uptake and results in a decrease in the plasma concentration of LDL-C. It has been well recognized that statins are associated not only with reduction of LDL-C levels but also with substantial reduction of the prevalence of coronary events. Clinical trials have confirmed that these agents reduce coronary events in subjects with and without coronary disease, reduce cardiovascular morbidity and mortality, and may even promote regression of atherosclerotic vascular lesions ${ }^{6-10}$ The benefits of statin therapy on primary and secondary prevention in patients with a wide range of LDL-C levels is therefore well established. The Pravastatin or Atorvastatin Evaluation and Infection Therapy (PROVE IT) ${ }^{11}$ and Treat to New Targets (TNT) ${ }^{12}$ studies showed that intensive lipid-lowering therapy significantly reduces the risk of cardiovascular disease events compared with moderate lipid-lowering therapy ( $\mathrm{p}=0.005$ and $\mathrm{p}<0.001$, respectively). These studies 


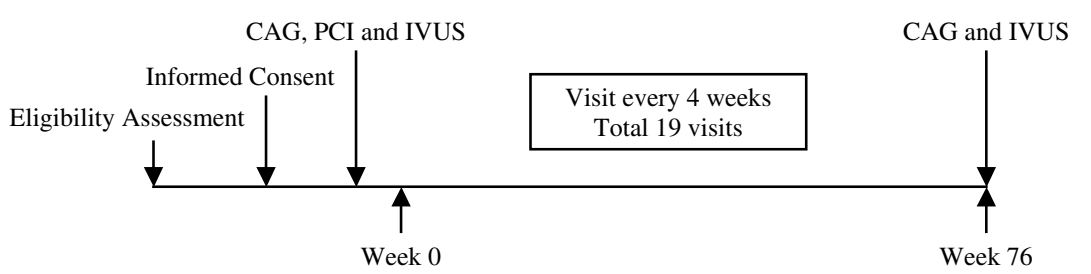

Fig 1. Flow chart showing the study timeline. CAG, coronary angiography; PCI, percutaneous coronary intervention; IVUS, intravascular ultrasound. would suggest that very intensive lipid lowering is required to induce regression of atherosclerosis.

The ability of statins to reduce progression of coronary atherosclerosis or to induce its regression has been evaluated by coronary angiography in a number of studies: MARS 13 CCAIT,14 The Multicenter Anti-Atheroma study (MAAS Investigators 1994), and Pravastatin Limitation of Atherosclerosis in the Coronary Arteries (PLAC-I) ${ }^{15}$ However, almost all the angiographic studies have revealed that the change in luminal parameters, such as the percent diameter stenosis and the minimal lumen diameter, was very subtle, although it was statistically significant. It was partially the vessel remodeling that masked the net change of plaque volume, and therefore, it has been recognized that direct plaque imaging might be more useful for assessing the effect of lipid-lowering drugs on the process of atherosclerosis.

Intravascular ultrasound (IVUS) is a modality that quantitatively represents atherosclerosis in vivo. IVUS enables accurate measurement of the lumen area, as well as atheroma size and distribution. The REVERSAL trial ${ }^{16}$ (Reversal of Atherosclerosis with Aggressive Lipid Lowering) and ASTEROID trial ${ }^{17}$ (A Study to Evaluate the Effect of Rosuvastatin on Intravascular Ultrasound-Derived Coronary Atheroma Burden) have successfully investigated the effects of statins on atherosclerosis. Most particularly, ASTEROID is the first study to clearly show a reversal of the atherosclerotic disease process in major clinical studies. This was a 24-month single-arm, blinded endpoint, multinational study conducted in 9 countries: Australia, Belgium, Canada, France, Italy, Netherlands, Spain, the United Kingdom, and the United States of America. For the primary efficacy parameter of the percentage atheroma volume, the median was $-0.79 \%(97.5 \%$ confidence interval $(\mathrm{CI})$, $-1.21 \%$ to $-0.53 \%)(\mathrm{p}<0.001$ compared with baseline $)$. This was accomplished with rosuvastatin $40 \mathrm{mg} /$ day, and reduced LDL-C by $53.2 \%$ and increased high-density lipoprotein (HDL)-C by $14.7 \%$. Rosuvastatin is the most effective of the new generation statins, and should enable more patients to achieve lipid goals with the starting dose ${ }^{18}$

In Japan, however, the beneficial effect of statin treatment on atherosclerotic lesions for 6 months after a coronary event was shown in the small, single-center, ESTABLISH Study 19 The subjects were randomized to atorvastatin (intensive lipid-lowering therapy) or control groups after percutaneous coronary intervention (PCI). LDL-C was significantly reduced by $41.7 \%$ in the atorvastatin group compared with an increase of $0.7 \%$ in the control group $(\mathrm{p}<0.001)$. Plaque volume was significantly reduced in the atorvastatin group $(13.1 \pm 12.8 \%$ decrease) compared with the control group $(8.7 \pm 14.9 \%$ increase; $\mathrm{p}<0.0001)$, even in patients with low baseline LDL-C $(<125 \mathrm{mg} / \mathrm{dl})$.

Based on a linear relationship identified between the decrease in LDL-C and the change in the luminal diameter of the coronary artery, it was suggested that at least $40 \%$ reduction in LDL-C is needed to arrest progression of the atherosclerotic process ${ }^{20}$ Birgelen et $\mathrm{al}^{21}$ reported possible suppression of progression of plaque (area) at LDL-C levels of $<75 \mathrm{mg} / \mathrm{dl}$. The ASTEROID trial suggested that treatment to LDL-C levels below currently accepted guidelines, such as NCEP ATP III and the Third Joint Task Force European guidelines, when accompanied by significant HDL-C increase, could produce regression of atherosclerosis in coronary disease patients. Recently, a meta-analysis has demonstrated that the pleiotropic effects of statins do not seem to contribute an additional cardiovascular risk reduction benefit beyond that expected from the degree of LDL-C lowering22 Therefore, there might be a fundamental 1-to-1 relationship between LDL-C levels and CHD events. However, the most relevant parameter to provoke significant change of plaque volume, especially for the Japanese, is still unknown: an absolute level of LDL-C or the magnitude of change in LDL-C?

\section{COSMOS Study}

The COSMOS study will be the first multicenter study especially in a Japanese population to evaluate the effects of rosuvastatin on regression of coronary atherosclerosis. Comparisons will be made between the measurements of atherosclerosis at the beginning vs the end of drug treatment. This study is a single-arm study. As placebo controlled trials of statins in this population are no longer ethically acceptable, a comparator group receiving either placebo or a less active statin will not be included in the COSMOS study. Moreover, current US and EU guidelines also recommend achieving more intensive target levels in very highrisk, secondary-prevention patients ${ }^{23}$ IVUS was selected to evaluate coronary artery atheroma volume as the primary endpoint because of the high sensitivity of this imaging method compared to coronary angiography (CAG).

The COSMOS study will provide new evidence and therapeutic standards for the prevention of CHD in Japan by controlling LDL-C levels with rosuvastatin.

\section{Study Design}

This will be a 76-week, open-label, multicenter study to evaluate the effect of rosuvastatin on coronary artery atheroma volume as measured by IVUS in patients with CHD.

Eligible patients will begin treatment with rosuvastatin $2.5 \mathrm{mg}$ once daily. The dosage will be increased by titration within the usual dose range with a treatment goal of lowering LDL-C below $80 \mathrm{mg} / \mathrm{dl}$ based on safety and the relationship between suppression of coronary artery plaque progression and LDL-C level in prior studies!1,15,16,19,21 If LDL-C levels are still $80 \mathrm{mg} / \mathrm{dl}$ or above after 4 weeks of treatment, the dosage may be increased up to a maximum of $20 \mathrm{mg} /$ day. If the investigator finds it necessary to reduce the dosage because of an excessive decrease in LDL-C $(<50 \mathrm{mg} / \mathrm{dl})$ or occurrence of adverse events, the dosage may be reduced again to the starting dose of $2.5 \mathrm{mg}$ once 
daily.

A total of 19 scheduled visits are planned during the course of this study. Subjects will attend follow-up visits every 4 weeks over 76 weeks after starting the treatment with rosuvastatin. IVUS and CAG will be performed at baseline and Week 76.

Prior to any study-related activities, all subjects will sign an informed consent form. This study is approved by the Institutional Review Board (IRB) or Independent Ethics Committee (IEC) of all of the participating centers (Fig 1). The planned duration is between October 2005 and October 2008.

\section{Patient Population}

All patients have to meet all of the inclusion criteria: aged 20-75 years undergoing CAG or PCI; serum cholesterol level either (a) untreated patients: LDL-C $\geq 140 \mathrm{mg} / \mathrm{dl}$ [calculated with Friedewald equation (triglyceride (TG) $<400 \mathrm{mg} / \mathrm{dl}$ ) or directly measured] or TC $\geq 220 \mathrm{mg} / \mathrm{dl}$, or (b) patients already treated with lipid-lowering agents: LDL-C $\geq 100 \mathrm{mg} / \mathrm{dl}$ [calculated with Friedewald equation (TG $<400 \mathrm{mg} / \mathrm{dl}$ ) or directly measured] or TC $\geq 180 \mathrm{mg} / \mathrm{dl}$; the patient must have at least 1 significant stenosis of $75 \%$ or more and be a candidate for PCI, and in addition to the candidate lesion for PCI, there must be at least 1 lesion $\leq 50 \%$ stenosis that can be imaged by IVUS.

Exclusion criteria are: (1) acute myocardial infarction within $72 \mathrm{~h}$ of the onset of the study, (2) heart failure of New York Heart Association class III or IV, (3) secondary hyperlipidemia, (4) administration of cyclosporine, (5) hemodialysis, (6) lesions requiring intervention, (7) left main coronary artery disease of $>50 \%$ stenosis, (8) uncontrolled hypertension (diastolic blood pressure $\geq 110 \mathrm{mmHg}$ or systolic blood pressure $\geq 200 \mathrm{mmHg}$ for all measurements during the screening period), (9) uncontrolled diabetes (hemoglobin A1c $\geq 9.5 \%$ ), (10) active liver disease or liver dysfunction with $\geq 2.5 \times \mathrm{ULN}$ (upper limit of the normal) of either alanine aminotransferase, aspartase aminotransferase or alkaline phosphatase, or $\geq 3.0 \mathrm{mg} / \mathrm{dl}$ of total bilirubin, (11) creatinine clearance $<30 \mathrm{ml} / \mathrm{min}$ or serum creatinine $>2.0 \mathrm{mg} / \mathrm{dl}$, (12) serum creatine kinase $>3 \times$ ULN, (13) short plaque lesions with a length less than $6 \mathrm{~mm}$.

\section{IVUS Examination}

IVUS will be used to examine lumen area, atheroma size and distribution at baseline and after 76 weeks of treatment. Investigators will be required to use the same imaging system with the same type of IVUS catheter for both the baseline and follow-up examinations: Clearview ${ }^{\circledR}$, Galaxy ${ }^{\mathrm{TM}}$ ultrasound system or Galaxy2 $2^{\mathrm{TM}}$ ultrasound system with the Atlantis ${ }^{\mathrm{TM}}$ SR Pro $240 \mathrm{MHz}$ imaging catheter (Boston Scientific, Natick, MA, USA). The images will be optimized under visual inspection by manipulating the system settings. The gain settings will be determined with the intention of maximizing image morphology without excessive dropout, not saturating adventitial intensity, and minimizing noise. The automated pullback device will be set with a speed of $0.5 \mathrm{~mm} / \mathrm{s}$. IVUS images will be recorded on superVHS (S-VHS) videotapes or Digital Video Disk plus ReWritable (DVD+RW) disk. The images will be logged and analyzed blind by 2 experienced technicians in the core lab.

\section{IVUS Analysis}

Plaque volume will be assessed by volumetric analysis with the echoPlaque2 system (Indec Systems Inc). Baseline and follow-up IVUS images will be reviewed side-by-side on a display, and the target segment selected. The target segment to be monitored will be determined in a non-PCI site ( $>5 \mathrm{~mm}$ proximal or distal to the PCI site) with a reproducible index such as side branches, calcifications, or stent edges.

\section{Endpoints}

The primary endpoint is the percent change in the plaque atheroma volume (target lesion length measured will be a minimum of $6 \mathrm{~mm}$ ) from baseline to Week 76 .

The secondary endpoints are actual volume changes and percentage changes in plaque area, in the vascular crosssectional lumen area and total vascular area from baseline to Week 76 at the same preselected coronary artery crosssection.

Percent changes from baseline to specified measurement time points in TC, LDL-C, very LDL-C (VLDL-C), HDLC, non-HDL-C (TC-HDL-C), TG and remnant like particle (RLP-C), apoprotein (Apo)A-I, ApoA-II, ApoB, lipoprotein (a) (Lp (a)), small dense LDL, HDL-2 and HDL-3 will also be calculated.

Changes in high sensitivity C-reactive protein from baseline to specified measurement time points will be calculated. RLP-C will be measured by the immunity adsorption method and ApoA-I, ApoA-II and ApoB by turbidimetric immunoassay. $\mathrm{Lp}$ (a) will be measured by latex-enhanced turbidimetric immunoassay and small dense LDL, HDL-2 and HDL-3 by the ultracentrifugation method. All laboratory measurements will be performed at a central clinical laboratory (SRL, Inc, Tokyo, Japan).

\section{Safety}

Safety will be observed throughout the study. Adverse events, subjective symptoms/objective findings, body weight, resting 12-lead ECG, chest X-ray, general blood tests (hematology, renal and liver functions, glucose metabolism), urinalysis, and vital signs (blood pressure, pulse) will be observed.

\section{Sample Size}

In the protocol, the assumptions used for power calculations require a sample size of 126 patients to provide $80 \%$ power (assuming a SD of 24.9\%) to detect a $6.3 \%$ difference in the primary endpoint with $2.5 \%$ type I error rate for a 1-sided test. It was therefore determined that the enrollment of 200 patients per treatment would provide an adequate number of patients.

\section{Analysis Population}

The primary analysis population for efficacy will comprise subjects who comply with the protocol and have IVUS data that can be evaluated at both baseline and Week 76. This analysis population is defined as a per-protocol set. A full analysis set, defined separately, will be used as the secondary analysis population.

\section{Efficacy Analysis}

The primary endpoint and secondary endpoints defined as percentage changes or changes from baseline will be summarized by mean, standard deviation, minimum, median and maximum, and then $95 \% \mathrm{CIs}$ will be calculated. The null hypothesis that percentage change or change from baseline is equal to 0 is tested by 1 -sample t-test. 


\section{Safety Analysis}

For safety evaluation, the numbers and prevalence of adverse events (including abnormal changes in physical values and clinical laboratory values) and the prevalence of adverse drug reactions (adverse events to which causality of rosuvastatin cannot be ruled out) will be calculated. Adverse events and adverse drug reactions will be summarized by type, severity, causality and duration of event.

\section{Study Organization}

Principal Investigator Masunori Matsuzaki, Division of Cardiology Medicine, Department of Medicine and Clinical Science, Yamaguchi University Graduate School of Medicine, Ube, Japan.

Steering Committee Tetsu Yamaguchi, Cardiovascular Center, Toranomon Hospital, Tokyo, Japan; Hiroyuki Daida, Department of Cardiology, Juntendo University School of Medicine, Tokyo, Japan.

Core Laboratory Satoshi Saito, Department of Advanced Medicine, Nihon University School of Medicine, Tokyo, Japan.

\section{Conclusion}

The COSMOS study will be the first multicenter study performed in a Japanese population using IVUS to evaluate the effects of rosuvastatin on regression of coronary atherosclerosis. We hope to show that intensive LDL-C lowering by rosuvastatin reduces coronary artery atheroma volume from baseline in diseased coronary segments.

\section{Acknowledgments}

This study is supported by AstraZeneca and Shionogi Co Ltd.

\section{References}

1. Kannell WB, Castelli WP, Gordon T, McNamara PM. Serum cholesterol, lipoproteins, and the reish of coronary heart disease: The Framingham Study. Ann Intern Med 1971; 74: 1-12.

2. Castelli WP. Epidemiology of coronary heart disease: The Framingham Study. Am J Med 1984; 76: 4-12.

3. Expert Panel on Detection, Evaluation, and Treatment of High Blood Cholesterol in Adults. Executive Summary of The Second Report of The National Cholesterol Education Program (NCEP) Expert Panel on Detection, Evaluation, and Treatment of High Blood Cholesterol In Adults (Adult Treatment Panel II). JAMA 1993; 269: 3015-3023.

4. Expert Panel on Detection, Evaluation, and Treatment of High Blood Cholesterol in Adults. Executive Summary of The Third Report of The National Cholesterol Education Program (NCEP) Expert Panel on Detection, Evaluation, and Treatment of High Blood Cholesterol In Adults (Adult Treatment Panel III). JAMA 2001; 285: 2486-2497.

5. Nakaya N, Kita T, Mabuchi H, Matsuzaki M, Matsuzawa Y, Oikawa $\mathrm{S}$, et al. Large-scale cohort study on the relationship between serum lipid concentrations and risk of cerebrovascular disease under lowdose simvastatin in Japanese patients with hypercholesterolemia: Sub-analysis of the Japan Lipid Intervention Trial (J-LIT). Circ J 2005; 69: 1016-1021.

6. Brown BG, Albers JJ, Fisher LD, Schaefer SM, Lin J-T, Kaplan C, et al. Regression of coronary artery disease as a result of intensive lipid-lowering therapy in men with high levels of apolipoprotein B. N Engl J Med 1990; 323: 1289-1298.

7. Downs JR, Clearfield M, Weis S, Whitney E, Shapiro DR, Beere PA, et al. Primary prevention of acute coronary events with lovastatin in men and women with average cholesterol levels: Results of AFCAPS/TexCAPS (Air Force/Texas Coronary Atherosclerosis Prevention Study). JAMA 1998; 279: 1615-1622.

8. Sacks FM, Pfeffer MA, Moye LA, Rouleau JL, Rutherford JD, Cole TG, et al. The effect of pravastatin on coronary events after myocardial infarction in patients with average cholesterol levels. $N$ Engl $J$ Med 1996; 335: $1001-1009$.

9. Shepherd J, Cobbe SM, Ford I, Isles CG, Lorimer AR, Macfarlane $\mathrm{PW}$, et al. Prevention of coronary heart disease with pravastatin in men with hypercholesterolemia. $N$ Engl J Med 1995; 333: $1301-$ 1307.

10. Yokoi H, Nobuyoshi M, Mitsudo K, Kawaguchi A, Yamamoto A. Three-year follow-up results of angiographic intervention trial using an HMG-CoA reductase inhibitor to evaluate retardation of obstructive multiple atheroma (ATHEROMA) Study. Circ J 2005; 69: $875-$ 883.

11. Cannon CP, Braunwald E, McCabe CH, Rader DJ, Rouleau JL, Belder R, et al. Intensive versus moderate lipid lowering with statins after acute coronary syndromes. $N$ Engl J Med 2004; 350: $1495-$ 1504.

12. LaRosa JC, Grundy SM, Waters DD, Shear C, Barter P, Fruchart JC, et al. Intensive lipid lowering with atorvastatin in patients with stable coronary disease. $N$ Engl J Med 2005; 352: 1425-1435.

13. Blankenhorn DH, Azen SP, Kramsch DM, Mack WJ, CashenHemphill L, Hodis HN, et al. Coronary angiographic changes with lovastatin therapy: The monitored atherosclerosis regression study (MARS). Ann Intern Med 1993; 119: 969-976.

14. Waters D, Higginson L, Gladstone P, Kimball B, LeMay M, Boccuzzi SJ, et al. Effects of monotherapy with HMG-CoA reductase inhibitor on the progression of coronary atherosclerosis as assessed by serial quantitative arteriography: The Canadian Coronary Atherosclerosis Intervention Trial (CCAIT). Circulation 1994; 89: $959-968$.

15. Pitt B, Mancini GB, Ellis SG, Rosman HS, Park J-S, McGovern ME. Pravastatin limitation of atherosclerosis in the coronary arteries (PLAC I): Reduction in atherosclerosis progression and clinical events. J Am Coll Cardiol 1995; 26: 1133-1139.

16. Nissen SE, Tuzcu EM, Schoenhagen P, Brown BG, Ganz P, Vogel $\mathrm{RA}$, et al. Effect of intensive compared with moderate lipid-lowering therapy on progression of coronary atherosclerosis: A randomized controlled trial. JAMA 2004; 291: 1071-1080.

17. Nissen SE, Nicholls SJ, Sipahi I, Libby P, Raichlen JS, Ballantyne $\mathrm{CM}$, et al. Effect of very high-intensity statin therapy on regression of coronary atherosclerosis. JAMA 2006; 295: $1556-1565$.

18. Jones PH, Davidson MH, Stein EA, Bays HE, McKenney JM, Miller E, et al. Comparison of the efficacy and safety of rosuvastatin versus atorvastatin, simvastatin, and pravastatin across dose (STELLAR trial). Am J Cardiol 2003; 93: 152-160.

19. Okazaki S, Yokoyama T, Miyauchi K, Shimada K, Kurata T, Sato H, et al. Early statin treatment in patients with acute coronary syndrome demonstration of the benefical effect on atherosclerotic lesions by serial volumetric intravascular ultrasound analysis during half a year after coronary event: ESTABLISH Study. Circulation 2004; 110: $1061-1068$.

20. Thompson GR. What targets should lipid-modulating therapy achieve to optimise the prevention of coronary heart disease? Atherosclerosis 1997; 131: 1-5.

21. von Birgelen C, Hartmann M, Mintz GS, Baumgart D, Schmermund A, Erbel R. Relation between progression and regression of atherosclerotic left main coronary artery disease and serum cholesterol levels as assessed with serial long-term ( $>$ or $=12$ months) follow-up intravascular ultrasound. Circulation 2003; 108: 2757-2762.

22. Robinson JG, Smith B, Maheshwari N, Schrott H. Pleiotropic effects of statins: Benefit beyond cholesterol reduction? A meta-regression analysis. J Am Coll Cardiol 2005; 46: 1855-1862.

23. Grundy SM, Cleeman JI, Merz CN, Brewer HB, Clark LT, Hunninghake DB, et al. Implications of recent clinical trials for the National Cholesterol Education Program Adult Treatment Panel III Guidelines. Circulation 2004; 110: 227-239.

\section{Appendix 1}

Investigators

S Fujii, Department of Cardiovascular Medicine, Graduate School of Medicine, Hokkaido University, Sapporo; N Funayama, Department of Cardiology, Hokkaido Cardiovascular Hospital, Sapporo; H Atsumi, Department of Internal Medicine, Yamagata Prefectural Shinjo Hospital, Shinjo; T Kubota, Department of Cardiology, Saitama Cardiovascular and Respiratory Center, Ohsato; T Ohba, Department of Internal Medicine, Chiba Hokusoh Hospital, Inba; T Takayama, Division of Cardiovascular Medicine, Department of Medicine, Nihon University School of Medicine, Tokyo; K Miyauchi, Department of Cardiology, Juntendo University School of Medicine, Tokyo; S Tani, Department of Cardiology, Nihon University Surugadai Hospital, Tokyo; S Ishiwata, Department of Cardiology, Cardiovascular Center, Toranomon Hospital, Tokyo; K Fukui, Department of Cardiovascular Medicine, Kanagawa Cardiovascular and Respiratory Center, Yokohama; T Matsushita, Department of Cardiovascular Medicine, Aichi Prefectural Cardiovascular and Respiratory Center, Ichinomiya; T Kondo, Department of Cardiology, Komaki City Hospital, 
Komaki; K Nishigaki, Department of Internal Medicine II, Gifu University Hospital, Gifu; H Matsuo, Department of Cardiology, Gifu Prefectural Gifu Hospital, Gifu; K Ueno, Department of Cardiology, Gifu Municipal Hospital, Gifu; T Kimura, Department of Cardiovascular Medicine, Kyoto University Hospital, Kyoto; A Kawamura, Division of Cardiology, National Cardiovascular Center, Suita; N Awata, Department of Cardiology, Osaka Medical Center for Cancer and Cardiovascular Diseases, Osaka; Y Kobayashi, Department of Internal Medicine and Cardiology, Osaka City University Medical School, Osaka; A Itoh, Department of Cardiology, Osaka City Central Hospital, Osaka; Y Nagai, Department of Cardiovascular Medicine, Rinku General Medical Center, Izumisano; Y Kihara, Department of Cardiovascular Medicine, Kobe General Hospital, Kobe; T Kawagoe, Department of Cardiovascular Medicine, Hiroshima Municipal Hospital, Hiroshima; T Hiro, Division of Cardiology Medicine, Department of Medicine and Clinical Science, Yamaguchi University Graduate School of Medicine, Ube; H Ogawa, Division of Cardiology, Tokuyama Central Hospital, Shunan; T Kawasaki, Department of Cardiovascular Medicine, Shinkoga Hospital, Kurume; T Honda, Cardiovascular Center,
Saiseikai Kumamoto Hospital, Kumamoto; M Nakamura, Department of Cardiology, Toho University Ohashi Medical Center, Tokyo; K Urasawa, Cardiovascular Center, Tokeidai Memorial Hospital, Sapporo; H Iida, Department of Cardiology, National Hospital Organization Hamada Medical Center, Hamada; S Ono, Department of Cardiology, Saiseikai Yamaguchi General Hospital, Yamaguchi; T Oda, Department of Cardiology, Shimane Prefectual Central Hospital, Izumo; S Minagoe, Department of Cardiology, National Hospital Organization Kagoshima Medical Center, Kagoshima; K Hibi, Cardiovascular Center, Yokohama City University Medical Center, Yokohama; N Kubo, Cardiovascular Division, Omiya Medical Center, Jichi Medical School, Saitama; Y Sakanoue, Department of Cardiology, Seichokai Fuchu Hospital, Izumi; M Kawashiri, Division of Cardiovascular Medicine, Graduate School of Medical Science, Kanazawa University, Kanazawa; H Shimomura, Division of Cardiology, Fukuoka Tokushukai Medical Center, Kasuga; T Fujita, Division of Cardiology, Sapporo-Higashi Tokushukai Medical Center, Sapporo; J Yajima, Department of Cardiology, The Cardiovascular Institute, Tokyo, Japan. 